\title{
Speed Modulated Social Influence in Evacuating Pedestrian Crowds
}

\author{
Hye Rin Lindsay Lee ${ }^{1}$. Abhishek Bhatia ${ }^{2,3}$. Jenný Brynjarsdóttir ${ }^{1,4}$. \\ Nicole Abaid ${ }^{5}$. Alethea Barbaro ${ }^{1}$. Sachit Butail ${ }^{6}$ \\ ${ }^{1}$ Case Western Reserve University, Department of Mathematics, \\ Applied Mathematics and Statistics, Cleveland, USA, \\ E-mail: hx1636@case.edu, alethea.barbaro@case.edu, jxb628@case.edu \\ ${ }^{2}$ Columbia University, Department of Computer Science, New York, USA, \\ ${ }^{3}$ Indraprastha Institute of Information Technology, Delhi, India \\ E-mail: ab4460@columbia.edu \\ ${ }^{4}$ University of Iceland, Faculty of Physical Sciences, Reykjavík, Iceland \\ ${ }^{5}$ Virginia Tech, Department of Mathematics, Blacksburg, USA \\ E-mail: nabaid@vt.edu \\ ${ }^{6}$ Northern Illinois University, Department of Mechanical Engineering, DeKalb, USA \\ E-mail: sbutail@niu.edu
}

Received: 13 November 2019 / Last revision received: 20 February 2020 / Accepted: 1 March 2020

DOI: $10.17815 / \mathrm{CD} .2020 .25$

\begin{abstract}
Evacuation is a complex social phenomenon with individuals tending to exit a confined space as soon as possible. Social factors that influence an individual include collision avoidance and conformity with others. While collision avoidance has been heavily focused on by the agent-based models used frequently to simulate evacuation scenarios, these models typically assume that all agents have an equal desire to exit the scene. It is more likely that, out of those who are exiting, some are patient while others seek to exit as soon as possible. Here, we experimentally investigate the effect of different proportions of patient (no-rush) versus impatient (rush) individuals in an evacuating crowd of up to 24 people. Our results show that a) exit speed changes significantly for individuals who otherwise tended to rush (or not rush) with both type of individuals speeding up in the presence of the other; and $b$ ) deviation rate, defined as the amount of turning, changes significantly for the rush individuals in the presence of no-rush individuals. We then seek to replicate this effect with Helbing's social force model with the twin purposes of analyzing how well the model fits experimental data, and explaining the differences in exit speed in terms of model parameters. We find that we must change the interaction parameters for both rush and no-rush agents depending on the condition that we are modeling in order to fit the model to the experimental data.
\end{abstract}


Keywords Evacuation $\cdot$ experiments $\cdot$ models $\cdot$ mixed intentions

\section{Introduction}

During emergencies in confined spaces, the exit presents a dwindling resource for evacuating individuals. Past emergencies that required evacuation have shown that a few seconds saved during egress can make considerable difference in outcomes [1]. Hence, one of the main goals for studying evacuation dynamics is to understand the role that individual, social, and environmental factors play in reducing the evacuation time at the cost of minimal injuries [2].

Experiments with evacuation are difficult to conduct because of the inherent safety concerns. Therefore, a large portion of the literature on evacuations relies on computer simulations to evaluate different scenarios [3]. These simulations in turn provide a basis for controlled experiments in the lab. Mathematical models that are used to simulate crowd evacuation can be broadly classified into physics based particle models that represent each individual as a finite sized particle which interacts with other particles via force fields $[4,5]$, cellular automata models that divides the space in which the individuals move into a grid, and populates each site within the grid based on the state of the agent [6-8], and macroscopic models that simulate the density and flow rates of a crowd as they exit a location. Such models have been used to evaluate the dependence of the flow rate of evacuating crowds on obstacles [9-11], as well as the influence of doorway width, placement, and whether it is open [10-12].

While the particle based models place a significant importance on the physical and social interactions that lead to avoidance and anticipation of collisions, recent experiments with actual crowds [13] and virtual reality platforms [14] have demonstrated the presence of a conforming social influence between individuals. Evacuation experiments by Nilsson \& Johansson [13] showed that social influence is higher from individuals that are closer and when information about the threat is ambiguous. In a study by Kinateder \& Warren [14] participants experienced active and passive bystanders who in turn influenced their tendency to evacuate a room, both in a real and virtual environment. Social influence is also likely to manifest when the evacuating crowd comprises individuals who have a different response to the emergency. In this context, Heliövaara et al. [15] model interaction between impatient and patient agents based on local conditions in the crowd. They show that an increased proportion of impatient agents can lead to bottlenecks within an otherwise safe egress. A relevant experimental study by Nicolas et al. [16] reported an increase in flow rate as the fraction of individuals who were asked to exit selfishly increased within an evacuating crowd.

In this study, we experimentally investigated the effect of impatient individuals in an exiting crowd. Impatient individuals corresponded to participants who were informed to leave the room quickly (rush) upon receiving a cue, as opposed to rest of the group who was instructed to simply leave the room (no-rush). Participants were requested to wear caps with color-coded symbols that were mapped to instructions. The number of rush participants were increased systematically in increments of twenty-five percent within a 
crowd of 21-24 individuals. Participants were tracked from an overhead camera as they exited the room. We made the following predictions: (i) the total time to exit for the participant crowd will decrease initially but then increase as the percentage of rush individuals in the crowd is increased; (ii) exit speed of individuals not rushing will be influenced by the presence of individuals who rush; and vice versa, the presence of participants instructed not to rush will influence the speed of individuals instructed to rush.

To analyze how the experimental results may affect individual perception, we fit an established model of crowd evacuation called the social force model [4] to experimental data. Specifically, we calibrated the model so that a subset of agents who correspond to rush agents evacuate with higher desired speeds in an arena that matched the dimensions of the experimental location. We then explored a statistically justified sample space for parameters corresponding to the strength and range of interaction force for avoiding collisions. Experimental results were compared to model results in terms of the Wasserstein distance between the distribution of exit speeds of agents from simulation and exit speeds of individuals from the experiment.

This paper is organized as follows: Sec. 2 describes the experimental setup and procedure and the data analysis. Sec. 3 reviews the social force model and details the modeling parameters and the design of the simulation experiments including the sampling approach and the Wasserstein metric for comparing and fitting simulated data to experiments. The experimental and simulation results are presented in Sec. 4 and discussed in Sec. 5.

\section{Experiments}

\section{Experimental setup}

The experimental arena consisted of a $8.18 \mathrm{~m}$ long $4.01 \mathrm{~m}$ wide $3.5 \mathrm{~m}$ high room with a single exit door. A wide-angle high-definition web camera (Microsoft LifeCam Cinema 720p, Microsoft Corporation, Redmond, WA, USA) filming at 30 frames per second in $1280 \times 720$ pixel resolution was mounted on a tripod at a height of $2.71 \mathrm{~m}$ in the corner of the room to record the evacuation experiments (Fig. 1). The participants were undergraduate and graduate students from IIIT-Delhi, aged between between 1835 years, and recruited via two methods: by placing an ad on public notice boards and by requesting them to participate as they had finished attending a lecture from a nearby classroom (more participants were recruited by the second method).

\section{Experimental procedure}

Prior to each trial, all participants gave a verbal informed consent regarding their participation in the experiments. This was obtained after the general procedure was explained to the group. All methods were performed in accordance with the relevant guidelines and regulations approved by the ethics committee at IIIT-Delhi. Each experimental trial consisted of a group of 21-24 individuals (see Supplementary document) exiting the room upon receiving a cue by the experimenter. Participants were requested to assemble within 


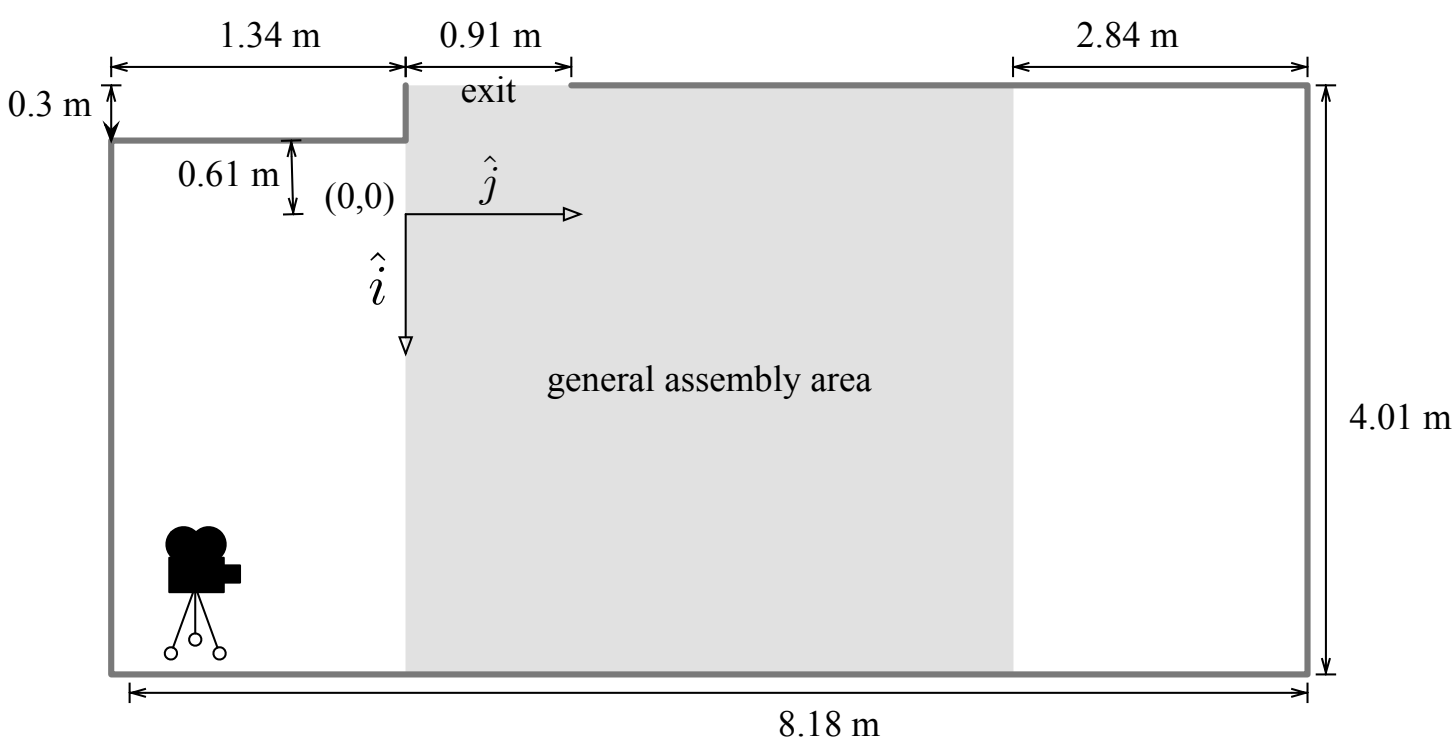

Figure 1 The experimental trials consisted of a group of participants evacuating a room. The general assembly area was a $4 \mathrm{~m}$ square within the room. The assembly area was unmarked and denotes an approximate region where the participants were assembled. The $(0,0)$ location marks the corner of a square tile near the door which was used to calibrate the camera with the two axes in the direction away from the exit $(\hat{i})$ and parallel to it $(\hat{j})$.

an approximately $4 \mathrm{~m} \times 4 \mathrm{~m}$ space near the door at the beginning of a trial. This resulted in an approximate density of 2.85 individuals $/ \mathrm{m}^{2}$ (Fig. 2). Each individual was given one of two specific instructions in writing on a piece of paper prior to the start of the experiment. These instructions, which were requested not to be shared with other participants, were to "leave the arena through the exit quickly" (rush) or to simply "leave the arena through the exit" (no-rush). These instructions were mapped to randomly assigned colorcoded white baseball caps that had one of five different symbols (circle, square, cross, $\mathrm{S}$, and I) in five different colors (red, green, blue, yellow, black) worn by each individual. The cap identifiers were used to map each participant to the paper instruction given to them prior to the experiment (Fig. 2). Upon receiving the cue, participants were instructed to "walk towards the exit and leave the arena, and keep walking until you are at least 6.1 meters (20 feet) away from the exit". Participants were advised not to talk or gesture to each other and avoid pushing nearby individuals.

The experimental conditions consisted of different proportions of the rush and no-rush individuals within a group. In particular, a total of five proportions were considered corresponding to rush (no-rush) percentages of 0(100), 25(75), 50(50), 75(25), and 100(0). Two trials were conducted per condition for all conditions except 50(50) for which only one trial was performed. Every group performed at most two trials, and no group was repeated for the same condition. A total of six different groups of participants were recruited for a total of approximately 140 individuals with three individuals common across all groups. Fig. 2 shows the start, middle and end of a sample trial of 100(0). 


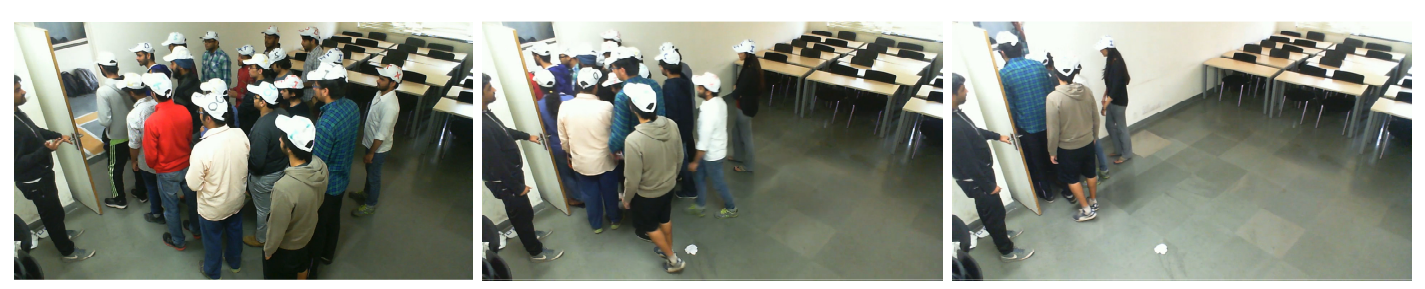

Figure 2 First, middle (approx.), and last frame from an experimental trial where all participants were requested to exit quickly, that is, rush(no-rush) percentage ratio was 100(0).

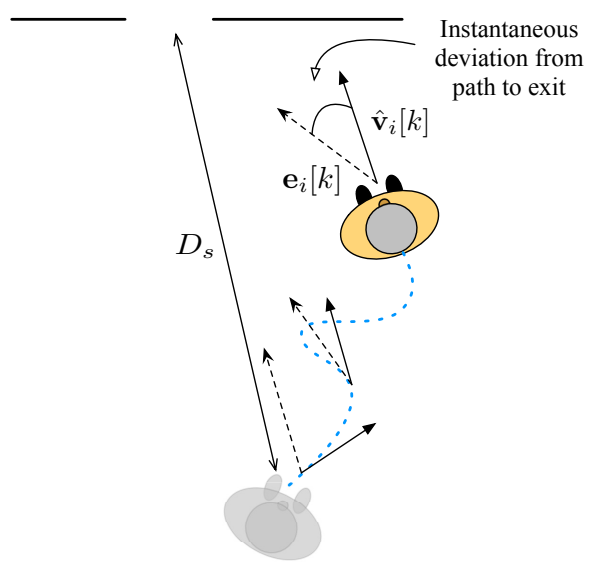

Figure 3 The shortest distance to exit $D_{s}$ from the starting position (light grey) and the instantaneous direction of motion $\hat{\mathbf{v}}_{i}[k]$ and exit direction $\mathbf{e}[k]$ at the current position (solid yellow) are shown.

\section{Experimental data analysis}

Participants' heads were tracked in each trial via provided uniform caps. Camera calibration was performed to estimate the camera intrinsic parameters of focal length and image center. Camera position and orientation during each experiment was estimated by optimally fitting a checkerboard pattern of known dimensions on the floor tiles of the room (see Supplementary document). With the assumption that the height of all participants was $1.65 \mathrm{~m}$ [17], we then computed the location of each tracked point in the video in $\mathrm{cm}$.

Participant movement was tracked using a multi-target tracking algorithm developed originally for fish behavior study [18]. Briefly, the input to the tracking algorithm was the centroid position in pixels of the baseball caps. The baseball caps were segmented after thresholding all but the white colors within a region of interest. The tracking algorithm estimated individual position and velocity in two dimensions by implementing a Kalman filter which assumed a constant velocity motion for an individual between two frames (1/30th of a second). Individual trajectories were verified and repaired to ensure that no track switches or breaks were recorded. Duplicate trajectories and those that did not belong to a person were removed from the dataset. To avoid sudden head movements and position errors introduced from manual repair, the trajectories were further smoothed using a moving window average with window size of 0.1 seconds ( 3 frames). 
Visual analysis of videos revealed that, in one of the trials, a participant pushed through the crowd and in another one, a participant did not move at all thereby becoming a stationary obstacle. These two trials were removed from further analysis leaving a total of seven trials with two trials for conditions 25(75) and 100(0) and one trial for the remaining.

To compare the movement of rush versus no-rush individuals across conditions, we compute the exit speed and deviation rate per individual. Specifically, we defined exit speed as $D_{s} / T_{e}$, where $D_{s}$ is the shortest distance to exit from the start position, and $T_{e}$ is the time to exit (Fig. 3); to compare the tendency of an individual to deviate from the instantaneous path to exit, we defined deviation rate as the average of the instantaneous deviation of direction of motion from exit direction calculated as $\left[\sum_{k} \cos ^{-1}\left(\hat{\mathbf{v}}_{i}[k]^{\mathrm{T}} \mathbf{e}[k]\right)\right] / T_{e}$, where $\hat{\mathbf{v}}_{i}[k]$ is the unit velocity vector at time step $k$ and $\mathbf{e}[k]$ is the instantaneous vector oriented towards the exit (Fig. 3).

To test our hypotheses, namely that individual behavior will change depending on the proportion of the crowd, we used a mixed effects model. Specifically, we separately compared the exit speed and deviation rate of each individual (response) for each instruction of rush and no-rush across proportions (fixed effect of the fraction of rush participants); to allow for dependence between behaviors within a group, trial number was modeled as a random effect. After verifying that the data was not distributed normally, post-hoc pairwise comparisons were performed using Wilcoxon rank sum test by considering all individual-level measurements for a condition as the response variable, and with significance set at $p<0.01$. Since the behavior that led to the rejection of the two trials may be considered as realistic, we performed all statistical analyses again by including the two rejected trials to investigate the amount by which this could affect our results. Further analysis was performed using a model-based approach where the experimental data was fit to an established pedestrian dynamics model described next.

\section{Modeling}

\section{Crowd motion model}

Simulation of crowd evacuation was performed using an agent based model, namely the social force (SF) model [4]. This method models the dynamics of each agent within a set of finite-sized agents by updating the interaction forces with the surroundings, obstacles, and other agents at every time step. To simulate an evacuation scenario, the agents are subjected to an additional goal force which is directed towards an exit.

The social force model [4] represents the total force on each agent $i$ as the sum of a goal force $\mathbf{f}^{g} \in \mathbb{R}^{2}$, sum of interaction forces with all other agents indexed by $j, \mathbf{f}_{i j} \in \mathbb{R}^{2}$, and interaction with the walls, $\mathbf{w}$, or obstacles $\mathbf{f}_{i w} \in \mathbb{R}^{2}$. The governing equation for agent $i$ is

$$
m_{i} \frac{d \mathbf{v}_{i}}{d t}{ }_{i}=m_{i} \mathbf{f}_{i}^{g}+\sum_{j=1, j \neq i}^{N} \mathbf{f}_{i j}+\sum_{w} \mathbf{f}_{i w}
$$


where the goal force is

$$
\mathbf{f}_{i}^{g}=\frac{v_{i}^{0} \mathbf{e}_{i}^{0}(t)-\mathbf{v}_{i}(t)}{\tau_{i}}
$$

and the interaction force is

$$
\mathbf{f}_{i j}=\left\{A_{i} e^{\frac{\left(r_{i j}-d_{i j}\right)}{B_{i}}}+k g\left(r_{i j}-d_{i j}\right)\right\} \mathbf{n}_{i j}+k g\left(r_{i j}-d_{i j}\right) \Delta v_{j i}^{t} \mathbf{t}_{i j} .
$$

Here, $v_{i}^{0}$ is the desired speed, $\mathbf{e}_{i}^{0}$ is goal direction and $\tau_{i}$ is the adaptation time that determines the time it takes for an agent to turn in a desired direction [19]; $A_{i}$ and $B_{i}$ are constants that determine the interaction strength and the size of interaction radius, $r_{i j}=r_{i}+r_{j}$ is the sum of agents' radii, $d_{i j}$ is relative distance between them; $\mathbf{n}_{i j}$ is a unit vector from $j$ to $i, \mathbf{t}_{i j}$ is the tangential direction, $\Delta v_{j i}^{t}$ is the tangential velocity difference, and $\kappa=3000$ and $k=750$ are some constants; $g(x)$ is the function that is zero when the pedestrians are not in contact $\left(d_{i j}>r_{i j}\right)$, else it is equal to the argument $x$. The wall interaction force $\mathbf{f}_{i w}$ has the same structure as $\mathbf{f}_{i j}$ with $j$ representing stationary agents that are unaffected by forces.

\section{Model parameters}

The spatial dimensions for simulating crowd evacuation has the exact same environment as the experiment. The arena consists of a $4 \mathrm{~m} \mathrm{x} 4.5 \mathrm{~m}$ grid with a $1.0 \mathrm{~m}$ wide exit placed at the left side of the grid. The arena in the simulations was made slightly larger to avoid physical overlap with the wall. The dimensions of the room were manually adjusted so that initial conditions did not collide with the boundary. Simulations are initialized by placing $N=21-24$ agents inside the grid by using both the initial positions of the participants from the experiment and observations of the video data. The size of an agent $d_{i}=2 r_{i}$ is sampled from a uniform distribution with mean $0.393 \mathrm{~m}$ and standard deviation 0.03 $\mathrm{m}$ based on data from an anthropometric study of Indian male workers [20]. To ensure that agents are not within colliding distance from each other at the start of the simulation, agent position is resampled until it is more than $H *$ (sum of the two agents' radii), where the factor $H=1.1$ is set by the code developed by Helbing et al. [4] which is a discrete time version of (Eq. 1). The agents are divided into rush and no-rush based on their desired speed $v_{i}^{o}$. These values were fitted to the exit speeds of rush and no-rush agents in the conditions 0(100) and 100(0) respectively. We tested wide ranges of values for $A$, $B$, and $v_{i}^{0}$ to fit the model to the experiment; this fitting process is discussed in the next subsection. The rest of the model parameters are set to default values as in Helbing et al. [4]. Model parameters are listed in Tab. 1.

We use an ensemble of one hundred simulations per parameter set, $\left[A_{\mathrm{R}}, B_{\mathrm{R}}, v_{\mathrm{R}}^{0}, A_{\mathrm{NR}}\right.$, $B_{\mathrm{NR}}, v_{\mathrm{NR}}^{0}$, where the subscripts R and NR denote the parameters for the rush and no-rush agents, respectively. Simulations are performed for each condition corresponding to the ratio of rush to no-rush agents in the experiment. The simulations are run until all agents exit the arena. 
Table 1 Parameters used for simulating crowd models

\begin{tabular}{lll}
\hline Parameter & Description & Value (unit) \\
\hline$m_{i}$ & mass & $80(\mathrm{~kg})$ \\
$\tau_{i}$ & adaptation time & $0.5(\mathrm{~s})$ \\
$A_{i}$ & interaction strength & {$[0,4000](\mathrm{N})$} \\
$B_{i}$ & characteristic length scale of interaction range & {$[0.0001,0.16](\mathrm{m})$} \\
$v_{i}^{0}$ & desired speed & {$[0,2]\left(\frac{\mathrm{m}}{\mathrm{s}}\right)$}
\end{tabular}

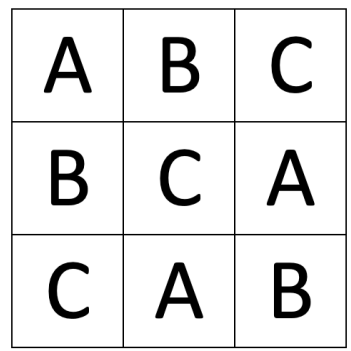

Figure 4 A 3 by 3 Latin Square.

\subsection{Simulation design}

Latin Hypercube Sampling One efficient method to sample points from a wide range of parameter values is called the Latin Hypercube Sampling. The idea of Latin Hypercube Sampling comes from Latin Square. In Fig. 4, we see that letters A through C are placed in each row and column of the square. A Latin Square ensures that each letter appears exactly once in each row and column. Latin Hypercube Sampling normally has a sampling region of the unit square, $[0,1]^{2}$. However, this can be extended to any value. Let us say that we want to sample $n$ points. We divide the region, $[0,1]$, into $n$ equally spaced intervals, $[0,1 / n], \ldots,[(n-1) / n, 1]$; which results in $n^{2}$ squares in the sampling region. Now, we fill these squares with the integers $1,2, \ldots, n$ to form a Latin square, and we select one of the integers at random. The value that resides in the selected integer's square become a Latin Hypercube Sampling of size $n$ [21]. As we saw in the Latin Square, this method ensures that points are spread evenly over the sampling space. We sample 600 parameter sets using Latin Hypercube Sampling. We then use those sets to simulate (Eq. 1) to fit the model to the experiment data.

Wasserstein Distance The metric we use to measure the closeness of fit between the experimental and simulation data is the Wasserstein 2-distance, also known as the Earth Mover's Distance. To visualize this distance, suppose we have two sand piles, A and B, with distinct shapes. The Wasserstein 2-distance between the two piles is the minimal amount of energy we would need to reshape and move the sand pile A so that it is exactly the sand pile B, or vice versa. The sand piles in our case represent the distributions of exit speeds from the experimental and simulation data. If two distributions are identical, the Wasserstein 2-distance between them is 0 . The farther apart and more different the 
distributions are, the larger the Wasserstein 2-distance becomes.

Although the Wasserstein 2-distance is hard to compute in higher dimensions, it is straightforward to calculate in one-dimension [22-24]. For a measure $\alpha$ on $\mathbb{R}$, its cumulative distribution function (CDF) from $\mathbb{R} \rightarrow[0,1]$ is defined for all $x \in \mathbb{R}$ as

$$
C_{\alpha}(x)=\int_{-\infty}^{x} d \alpha
$$

Its psuedo-inverse is then defined to be $C_{\alpha}^{-1}:[0,1] \rightarrow \mathbb{R} \cup\{-\infty\}$ such that, for all $r \in[0,1]$,

$$
C_{\alpha}^{-1}(r)=\min _{x}\left\{x \in \mathbb{R} \cup\{-\infty\}: C_{\alpha}(x) \geq r\right\}
$$

The following equivalence then holds for the Wasserstein 2-distance:

$$
W_{2}(\alpha, \beta)^{2}=\left\|C_{\alpha}^{-1}-C_{\beta}^{-1}\right\|_{L^{2}([0,1])}^{2}=\int_{0}^{1}\left|C_{\alpha}^{-1}(r)-C_{\beta}^{-1}(r)\right|^{2} d r
$$

We will refer to the Wasserstein 2-distance as simply the Wasserstein distance for the rest of the paper.

When using the Wasserstein distance in our calculations, we need to consider that the experimental subjects who were told to leave the room quickly (rush) have a distribution of exit speeds, and so do the experimental subjects who were told to simply leave the room (no-rush). In order to account for this, we use the Wasserstein distance to compare the distribution of the experimental rush subjects to the distribution produced by the simulated rush agents in one of the ensemble of runs. We then average this distance over the ensemble of runs and consider this to be the Wasserstein distance for the rush agents. We then repeat the process with the experimental subjects who were not given this instruction and the no-rush agents in our simulations. To determine the distance between the experiment and the simulation with a given set of parameter values, we then weight the rush Wasserstein distance and the no-rush Wasserstein distance according to the proportion of rush and no-rush subjects in the experiment. For example, in the case of 75(25), i.e. the condition with $75 \%$ rush and $25 \%$ no-rush, the overall Wasserstein distance is calculated as

$$
\mathrm{WD}=\frac{3}{4}(\text { Rush WD })+\frac{1}{4}(\text { No rush WD })
$$

where WD is the Wasserstein distance that we are trying to minimize.

\section{Parameter Selection}

To determine the best-fitting parameter set, $\left[A_{\mathrm{R}}, B_{\mathrm{R}}, v_{\mathrm{R}}^{0}, A_{\mathrm{NR}}, B_{\mathrm{NR}}, v_{\mathrm{NR}}^{0}\right]$, for the each experimental condition, we proceed as follows. First, we fix the desired speeds of the endpoints of the experimental conditions, i.e. the all rush case, 100(0), and all no-rush case, 0(100). In order to do so, we use Latin Hypercube Sampling to draw 600 points in the six-dimensional parameter space, in the range between the lowest and highest permissible value for each parameter. These values can be found in Tab. 1. After simulating each 

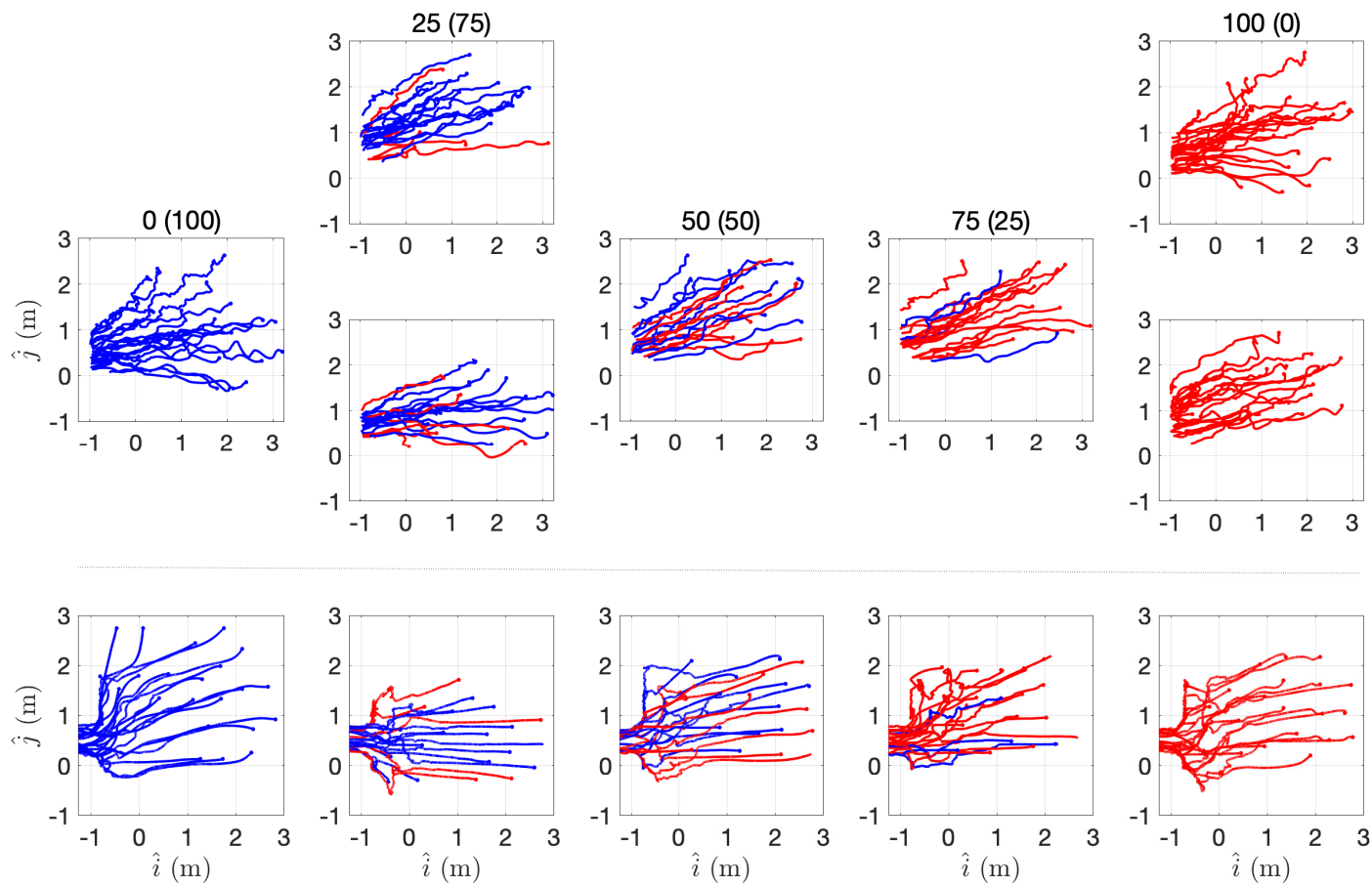

Figure 5 (Top) Raw trajectory data of rush (red) and no-rush individuals (blue) for all experimental trials considered. Titles show rush (no-rush) percentage. (Bottom) Raw trajectory data of rush (red) and no rush (blue) agents for one simulation of each condition, using the best-fit parameter values. From left to right, the represented conditions are: $0(100), 25(75), 50(50), 75(25)$, and $100(0)$. The door in each case is located between $(-1,0)$ and $(-1,1)$.

parameter set 100 times, we use the ensemble average of the 100 Wasserstein distances for each set to determine which one gives the closest fit, as determined by the minimum average Wasserstein distance. The best-fitting desired speeds for the all rush case and the no-rush case become the fixed desired speed for each rush and no-rush agent over all the conditions, respectively. The reason behind fitting the best desired speeds and fixing them for the endpoints is to have uniform desired speeds for all rush or no-rush agents regardless of the experimental conditions, since our assumption was that the desired speed of the participant should be determined by the strength of their motivation to exit the room. Next, we redraw 600 points again using Latin Hypercube Sampling for four remaining parameters, $\left[A_{\mathrm{R}}, B_{\mathrm{R}}, A_{\mathrm{NR}}, B_{\mathrm{NR}}\right]$, now keeping $v_{\mathrm{R}}^{0}$ and $v_{\mathrm{NR}}^{0}$ fixed at the values determined by the fitting the all rush and no-rush experiments. We redraw to eliminate the dependency of desired speed from the previous parameter sets. We again find the minimum ensemble average of the 100 Wassertein 2-distances to determine the best fitting parameter set. 

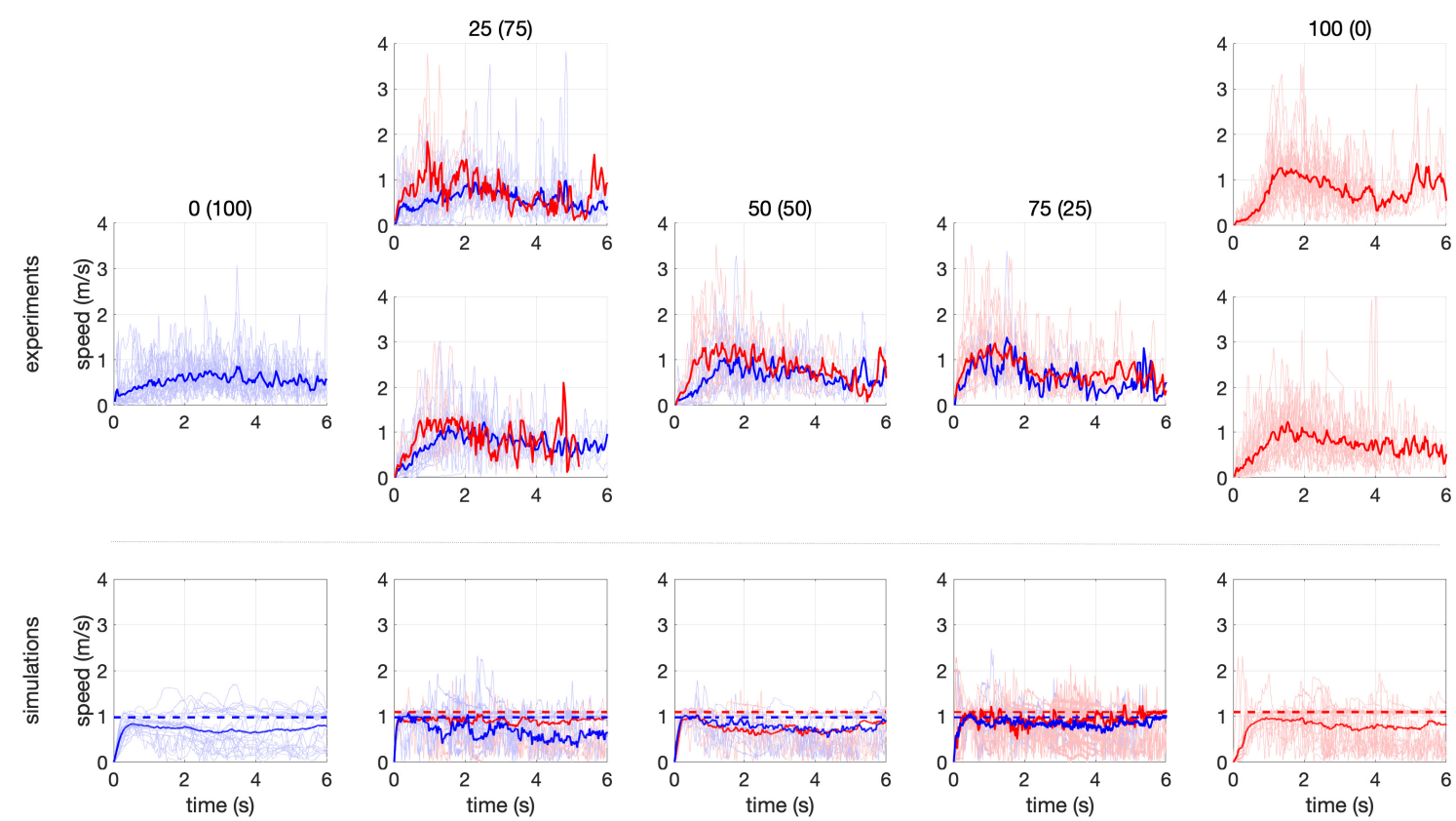

Figure 6 (Top) Instantaneous speeds of rush (red) and no-rush individuals (blue) for all experimental trials considered. Darker lines show the instantaneous speed averaged across participants of a particular type. (Bottom) Instantaneous speed of all simulation agents of each condition, using one run with best-fit parameter values. The red lines are rush agents, and the blue lines are no rush agents. The bold lines are the average instantaneous speed of each participant type, and the dotted lines are the desired speed. From left to right, the represented conditions are: $0(100)$, 25(75), 50(50), 75(25), and 100(0).

\section{Results}

\section{Experimental results}

The top of Fig. 5 shows the raw trajectories of participants in each of the trials considered for analysis. The trajectories are directed towards the door located between $(-1,0)$ and $(-1,1)$ near the corner of the room. In this and all subsequent figures, variables for rush are depicted in red, and variables for no rush are depicted in blue. The top of Fig. 6 shows instantaneous speeds of all rush and no-rush individuals in each of the trials as they exit the room. Almost all the trials showed a sudden increase in speeds during the first 3 seconds before settling down to a lower value. (The bottom portion of Fig. 5 and 6 are referred to later in the text.)

The time to exit for the first eighteen participants is shown on the left in Fig. 7. (The right portion of this figure is referred to later.) The time to exit for eighteen individuals was longest for the 0(100) group at 7.7 seconds with all no-rush participants, while the shortest time to exit at 4.7 seconds was recorded for one of the 100(0) group.

Fig. 8 compares the exit speeds of participants between conditions. Our results showed that for both rush and no-rush participants, exit speeds depended on the fraction of rush participants (Tab. 2). Whereas for rush individuals, the exit speed decreased with increase 

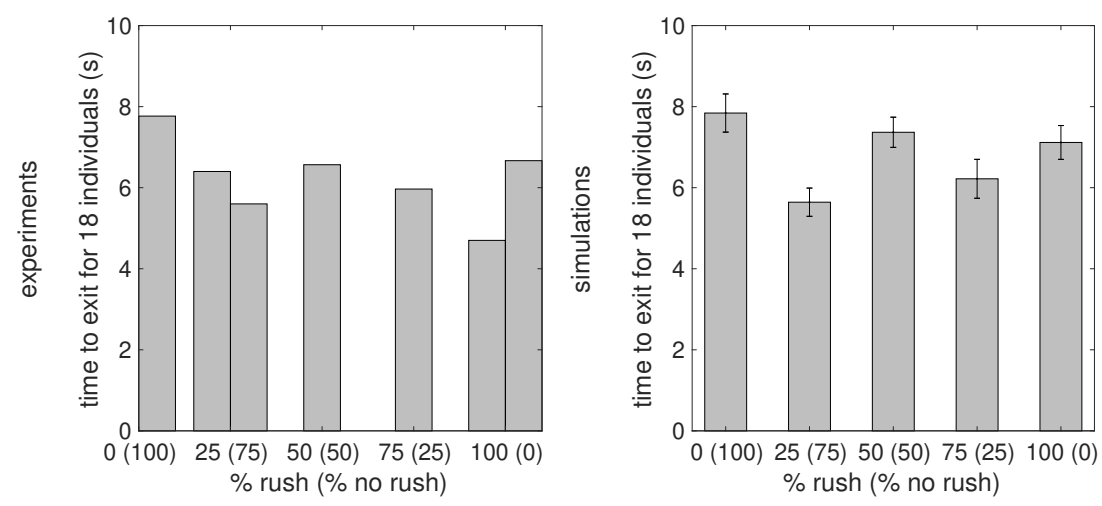

Figure 7 (Left) Time to exit in seconds for the first eighteen individuals in each group across different conditions of the experiment. For conditions 25(75) and 100(0), where multiple trials were analyzed, the adjacent bars show value for each trial. (Right) Average \pm standard deviation of time to exit in seconds for 18 agents in simulations with best-fit parameters across different conditions.

\begin{tabular}{llrrrrrr}
\hline & Name & Estimate & SE & DF & $p$ & Lower Bound & Upper Bound \\
\hline \multirow{2}{*}{ exit speed (Rush) } & Intercept & 1.042 & 0.08361 & 82 & $<0.01$ & 0.87563 & 1.2083 \\
\cline { 2 - 8 } & fraction of rush & -0.35526 & 0.10078 & 82 & $<0.01$ & -0.55575 & -0.15477 \\
\hline \multirow{2}{*}{ exit speed (No-Rush) } & Intercept & 0.55253 & 0.034262 & 70 & $<0.01$ & 0.48419 & 0.62086 \\
\cline { 2 - 7 } & fraction of rush & 0.21451 & 0.10939 & 70 & 0.05 & -0.0036745 & 0.43269 \\
\hline
\end{tabular}

Table 2 Linear mixed effects model investigating the effect of fraction of rush participants on the exit speed of rush and no-rush participants. Trial number was modeled as random effect.

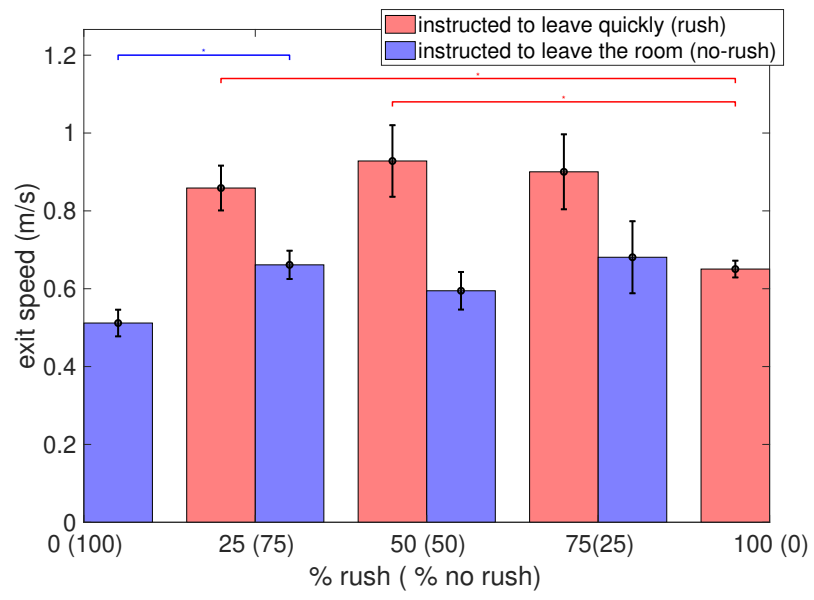

Figure 8 Exit speeds of individual participants across different conditions of the experiment. Overhead bars denote significant difference $(p<0.01)$ in post-hoc comparisons, for no-rush (blue) and rush (red) participants. Error bars denote mean \pm standard error across all participants for a condition. 


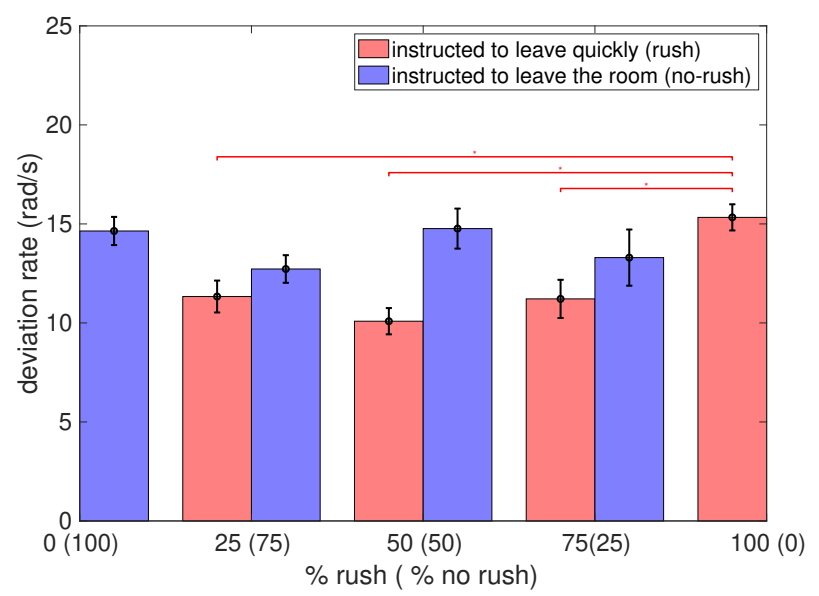

Figure 9 Deviation rates of individual participants across different conditions of the experiment. Overhead bars denote significant difference $(p<0.01)$ in post-hoc comparisons for rush (red) participants. Error bars denote mean \pm standard error across all participants for a condition.

in fraction of rush participants, for no-rush individuals, exit speed increased marginally as the group constituted more rush participants. (The coefficients for fixed effects remained within a hundredth decimal place of each other with different smoothing window sizes of two and five steps.) Post-hoc comparisons showed that the exit speed of no-rush individuals was significantly less in a purely no-rush group at $0.51 \mathrm{~m} / \mathrm{s}$ than at $0.65 \mathrm{~m} / \mathrm{s}$ when they comprised $75 \%$ of the group. For rush, post-hoc comparisons showed a drop in exit speed from $0.86 \mathrm{~m} / \mathrm{s}$ and $0.92 \mathrm{~m} / \mathrm{s}$ at when more than $50 \%$ of the group consisted of no-rush individuals to $0.65 \mathrm{~m} / \mathrm{s}$ in a purely rush group.

\begin{tabular}{llrrrrrr}
\hline & Name & Estimate & SE & DF & $p$ & Lower Bound & Upper Bound \\
\hline \multirow{2}{*}{ deviation rate (Rush) } & Intercept & 8.0405 & 1.3496 & 82 & $<0.01$ & 5.3558 & 10.725 \\
\cline { 2 - 7 } & fraction of rush & 6.7214 & 1.6268 & 82 & $<0.01$ & 3.4853 & 9.9576 \\
\hline \multirow{2}{*}{ deviation rate (No-Rush) } & Intercept & 13.966 & 0.6684 & 70 & $<0.01$ & 12.633 & 15.299 \\
\cline { 2 - 7 } & fraction of rush & -1.113 & 2.1341 & 70 & 0.60 & -5.3694 & 3.1435 \\
\hline
\end{tabular}

Table 3 Linear mixed effects model investigating the effect of fraction of rush participants on the deviation rate of rush and no-rush participants. Trial number was modeled as random effect.

Fig. 9 shows the deviation rates between conditions and Tab. 3 shows the results of the linear mixed-effects model. Our results showed that while for rush individuals, deviation rate increased strongly with the fraction of rush participants in the group, the same was not true for no-rush. (The coefficients for fixed effects with different smoothing window sizes of two and five steps remained within the confidence intervals of the coefficient with smoothing window size of three steps.) Post-hoc comparisons for rush participants showed that the deviation rate of rush participants in the purely rush group was significantly more than when no-rush participants were present in the group.

Including the two rejected trials as part of the analyses (see Supplementary document): (i) did not affect the negative dependence of exit speed of rush individuals on the fraction 


\begin{tabular}{c|cccccc}
\hline Conditions & WD Rush & WD No-Rush & A Rush & B Rush & A No-Rush & B No-Rush \\
\hline $100(0)$ & 0.0430 & NA & 2600.8161 & 0.0122 & NA & NA \\
& 0.0432 & NA & 772.2899 & 0.0146 & NA & NA \\
& 0.0434 & NA & 3939.7681 & 0.0110 & NA & NA \\
& 0.0439 & NA & 3119.1939 & 0.0117 & NA & NA \\
& 0.0442 & NA & 1960.1753 & 0.0127 & NA & NA \\
\hline $75(25)$ & 0.2752 & 0.1204 & 2532.2087 & 0.0021 & 2884.5443 & 0.0264 \\
& 0.3087 & 0.1225 & 2975.4024 & 0.0045 & 1837.2579 & 0.0327 \\
& 0.3101 & 0.1333 & 117.1384 & 0.0042 & 1976.2609 & 0.0338 \\
& 0.3318 & 0.1060 & 2858.4812 & 0.0083 & 3301.1246 & 0.0019 \\
& 0.3353 & 0.1071 & 1073.3886 & 0.0074 & 1251.6853 & 0.0021 \\
\hline $50(50)$ & 0.2686 & 0.0922 & 2057.3642 & 0.0075 & 1662.8214 & 0.0113 \\
& 0.2635 & 0.1051 & 210.2434 & 0.0058 & 3730.4057 & 0.0059 \\
& 0.2372 & 0.1329 & 1056.9571 & 0.0011 & 27.6546 & 0.0600 \\
& 0.2543 & 0.1172 & 1073.3886 & 0.0074 & 1251.6853 & 0.0021 \\
& 0.2940 & 0.0787 & 1948.6517 & 0.0086 & 8.6261 & 0.0578 \\
\hline $25(75)$ & 0.1924 & 0.1111 & 1073.3886 & 0.0074 & 1251.6853 & 0.0021 \\
& 0.1965 & 0.1168 & 2858.4812 & 0.0083 & 3301.1246 & 0.0019 \\
& 0.2117 & 0.1267 & 2723.7030 & 0.0136 & 3716.4583 & 0.0014 \\
& 0.2251 & 0.1300 & 2199.6064 & 0.0064 & 1741.9421 & 0.0045 \\
& 0.2435 & 0.1472 & 210.2434 & 0.0058 & 3730.4057 & 0.0059 \\
\hline $0(100)$ & NA & 0.0497 & NA & NA & 3.3546 & 0.1095 \\
& NA & 0.0598 & NA & NA & 8.6261 & 0.0578 \\
& NA & 0.0649 & NA & NA & 27.6546 & 0.0600 \\
& NA & 0.0652 & NA & NA & 417.2479 & 0.0186 \\
& NA & 0.0657 & NA & NA & 356.6604 & 0.0202 \\
\hline
\end{tabular}

Table 4 Top 5 parameter sets are given for all the experimental conditions. The Wasserstein distance is abbreviated as 'WD' in the table. From top to bottom, the order goes from the best to worst by rows for each cell.

of rush participants; (ii) removed the marginal dependence of the exit speed of no-rush individuals on fraction of rush participants; and (iii) weakened the dependence of deviation rate of rush individuals on fraction of rush participants. Post-hoc analyses with rejected trials retained all the pairwise comparisons found significant without such trials except that for deviation rate of rush participants between 100(0) and 75(25).

\section{Model results}

In Tab. 4, we can see the sets of parameter values for the simulations with the lowest five Wasserstein distances, together with the Wasserstein distance taken only between the experimental and simulated rush agents and the Wasserstein distance taken only between the experimental and simulated no-rush agents. We can observe the effect of weighing 

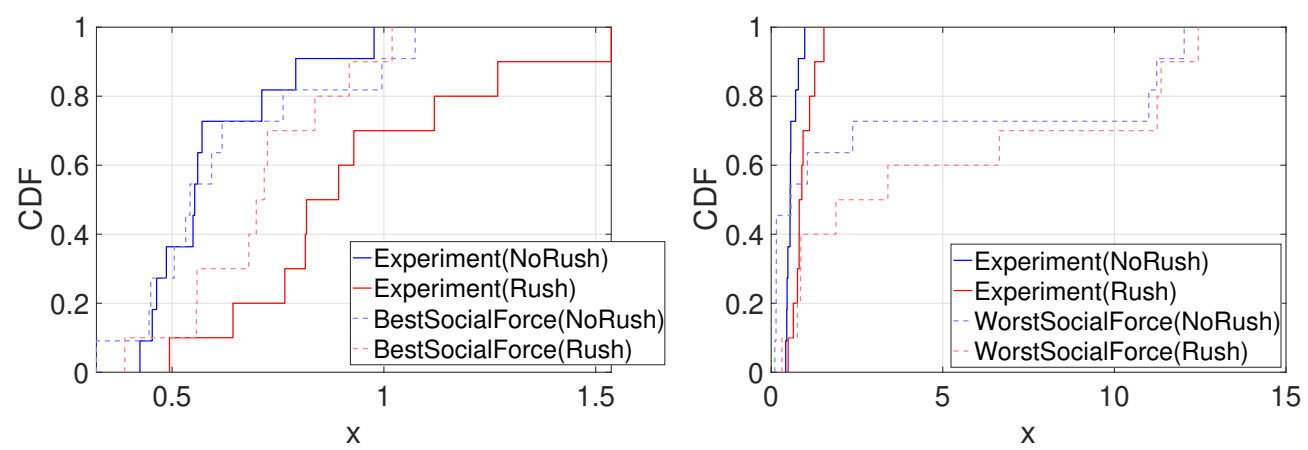

Figure 10 The cumulative distribution functions of the exit speed for the 50(50) condition. In both figures, the blue represents no-rush conditions, and the red represents rush conditions. The experimental CDF are shown as dark solid lines. The CDFs for one run using the parameter values giving the minimum Wasserstein distance is shown in light dashed lines (left), and the CDFs for a simulation performed using the parameters producing the maximum Wasserstein distance is in light dashed lines (right). In this case, the minimum Wasserstein distance is 0.1804 , whereas the maximum Wasserstein distance is 1.7029 .

the Wasserstein distance so that it considers how many agents of each type there are in the experimental condition (see Eq. 4) in condition 75(25), where the Wasserstein distance taken between the experimental and simulated no-rush agents is lower than that between the experimental and simulated rush agents.

The Wasserstein distance can be visualized in one dimension using the cumulative distribution function (CDF). In Fig. 10, we can see the CDFs for the exit speed of the experimental no-rush subjects for the 50(50) condition in dark blue, and the experimental rush subjects in dark red. A simulation using the parameters producing the lowest Wasserstein distance was performed, and the resulting CDFs of the exit speed of the no-rush and rush agents are shown in the left plot. The plot on the right shows the CDFs for a simulation performed using the parameters producing the maximal Wasserstein distance. As we can observe from the figure, the simulated CDFs for the minimum Wasserstein distance are much closer to the experimental CDFs than are the simulated CDFs for the maximum Wasserstein distance. In fact, the Wasserstein distance computed from the plot on the left is 0.1804 , while it is 1.7029 on the right.

It is helpful to view the Wasserstein distance as a function of the parameter values, in order to get a clearer idea of how $A_{\mathrm{NR}}, B_{\mathrm{NR}}, A_{\mathrm{R}}$, and $B_{\mathrm{R}}$ affect the fit of the model. In Tab. 5, we plot each of the sampled points in parameter space with a color corresponding to the Wasserstein distance between experiment and the simulation. This is then projected onto the plane spanned by $A_{\mathrm{NR}}$ and $B_{\mathrm{NR}}$ in the middle column, and $A_{\mathrm{R}}$ and $B_{\mathrm{R}}$ in the column on the right. Blue corresponds to a low Wasserstein distance, while red corresponds to a high Wasserstein distance. The five best parameter sets are identified by surrounding the point with a magenta star. The best fit is surrounded with a magenta star, while the other four are surrounded with magenta diamonds. For most of the plots, one can observe a rainbow-type pattern emerging, with the best fits corresponding to low $B_{\mathrm{NR}}$ and $B_{\mathrm{R}}$ values, and the worst fits occurring for high values of $B_{\mathrm{NR}}$ and $B_{\mathrm{R}}$. It appears that the model is not sensitive at all to changes of parameter $A$ although in some figures, smaller $A_{\mathrm{NR}}$ or 


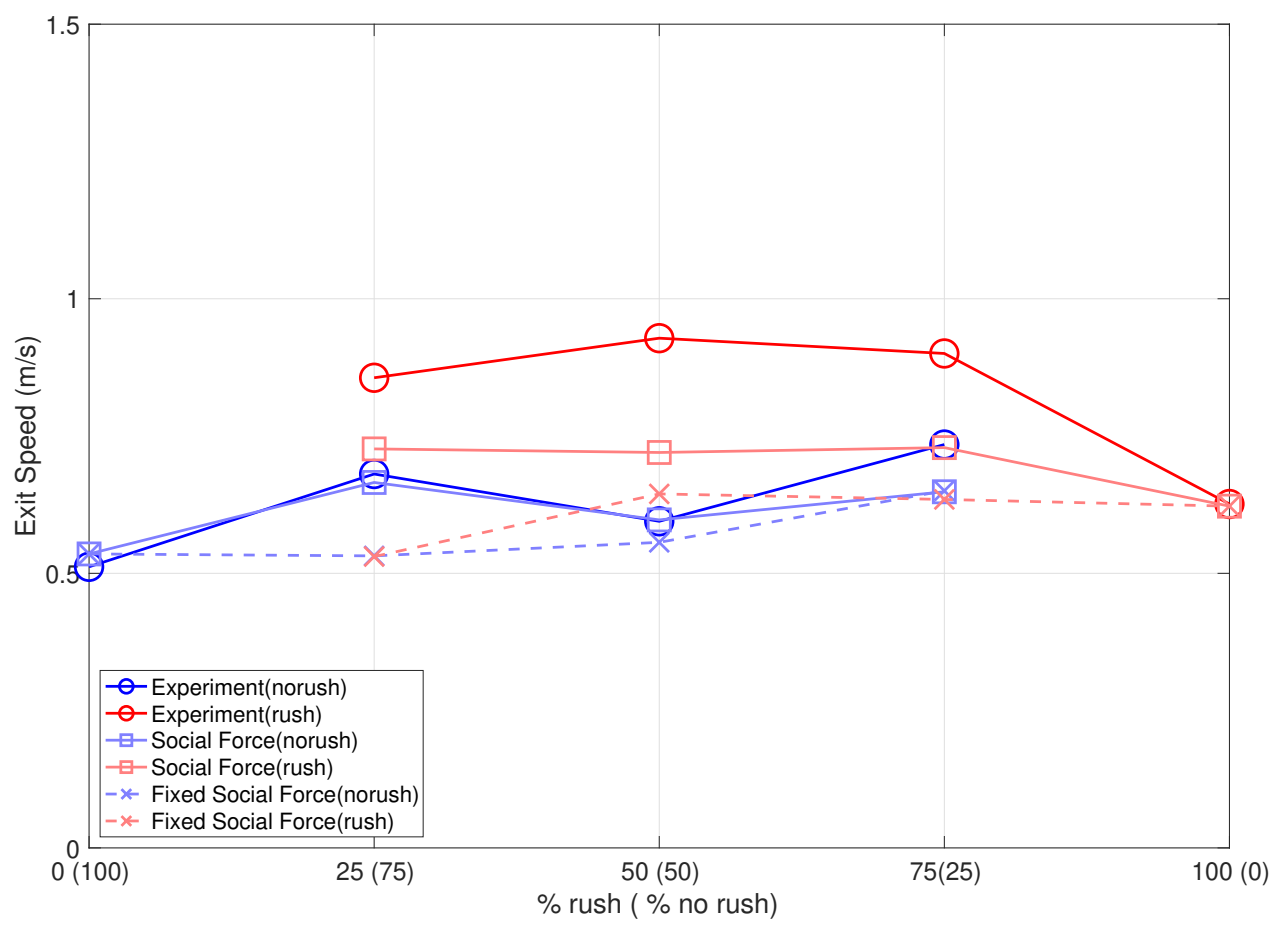

Figure 11 The exit speed of experiment (darker lines) and simulation (lighter lines). The dashed lines with ' $X$ ' markers are the exit speed of simulation with fixed A/B rush and no-rush across the all conditions.

$A_{\mathrm{R}}$ is preferred.

We found the best-fit parameter set for each condition from the sets proposed by the Latin Hypercube Sampling by minimizing the Wasserstein distance between the experimental run and the simulations, see Sec. 3.1. In Fig. 11, we show the exit speed of the no-rush and rush experimental subjects and plot this in solid dark blue and red, respectively. We then plot the results from the ensemble of best-fit simulations in solid light blue and red, as well. For comparison purposes, the dotted light red and blue lines show the exit speeds if we fix the $A_{\mathrm{NR}}$ and $B_{\mathrm{NR}}$ values throughout to be the best fit from the $0(100)$ condition, and we fix $A_{\mathrm{R}}$ and $B_{\mathrm{R}}$ throughout to be the best fit from the 100(0) condition. It is clear from this figure that, in order to attain the curves that we see experimentally, we must fit all four parameters for each condition independently.

To further explore the effects of the parameters on the model, we turn to Fig. 12 and 13. On the left of Fig. 12, we plot the best five $A_{\mathrm{NR}}$ in blue and $A_{\mathrm{R}}$ in red, with the top fit highlighted as a darker line. On the right, we do the same for the best five $B_{\mathrm{NR}}$ and $B_{\mathrm{R}}$. The range of the y-axis corresponds to the chosen parameter range. In Fig. 13, we similarly show the best parameter fits, but this time showing all parameters within a tolerance of 0.28 in Wasserstein distance. The best-fit $A$ parameters are plotted in the left column, and the $B$ parameters in the right column, with red indicating rush parameter values and blue indicating no-rush parameter values. From both plots, it becomes clear that the best-fit $A_{\mathrm{NR}}$ and $A_{\mathrm{R}}$ are distributed throughout the sampling range, while the best-fit $B_{\mathrm{NR}}$ and $B_{\mathrm{R}}$ clearly prefer the bottom of their range with an exception for $B_{\mathrm{NR}}$ of $0(100)$. 


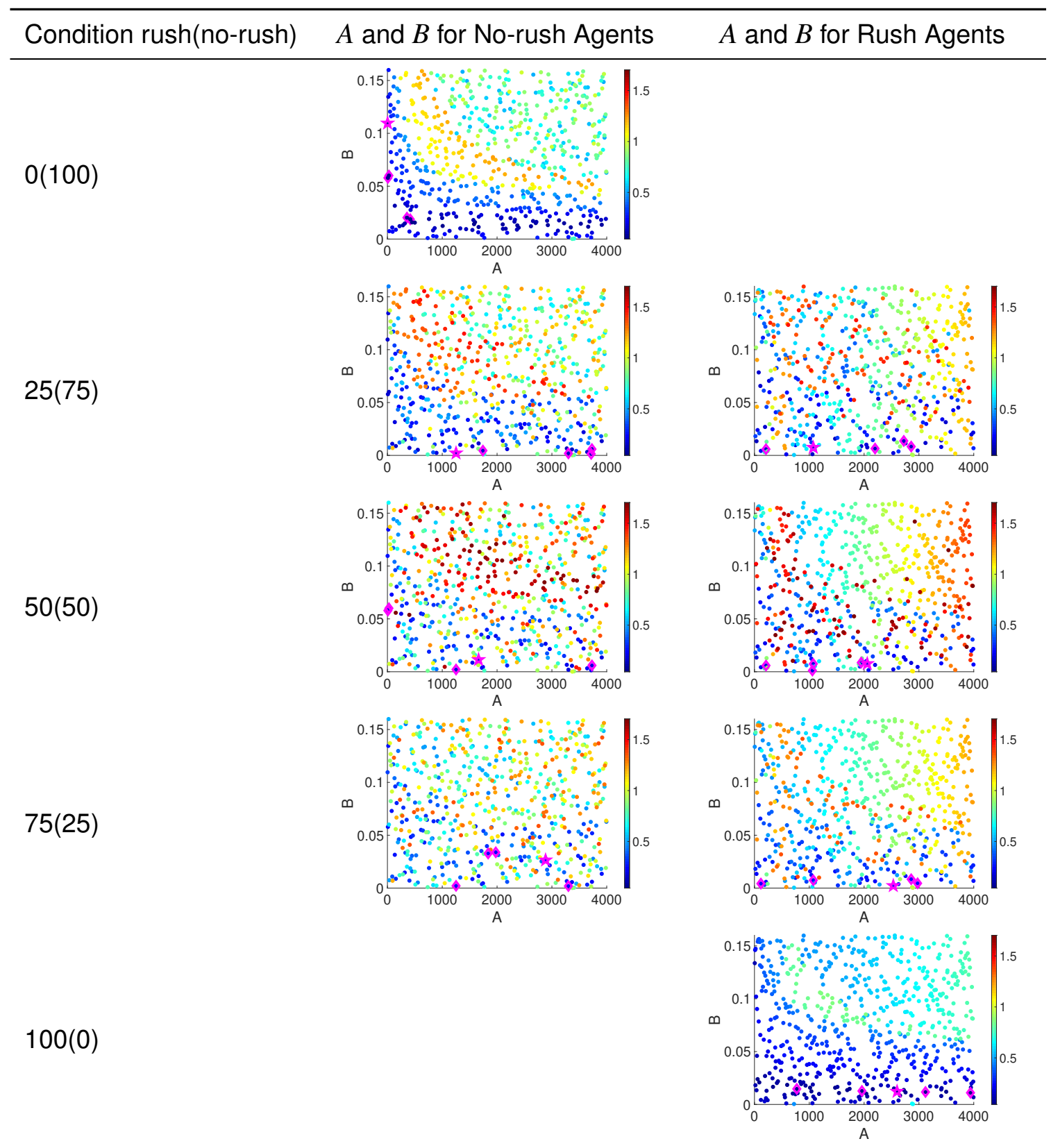

Table 5 Results from simulations of parameter sets given by Latin Hypercube Sampling, plotted in parameter space. Here, we plot the Wasserstein distances as a colored circle at the point in parameter space where it was run. The blue schemes represent lower Wasserstein distance values, whereas the red schemes represent higher values. The best parameter sets are filled with a magenta star, and the next top four parameter sets are filled with a magenta diamond. Since parameter space is four-dimensional, consisting of $\left[A_{\mathrm{R}}, B_{\mathrm{R}}, A_{\mathrm{NR}}, B_{\mathrm{NR}}\right]$, we look at the Wasserstein Distances projected onto parameter space for the no-rush agents on the left, and projected onto the rush agents on the right. 

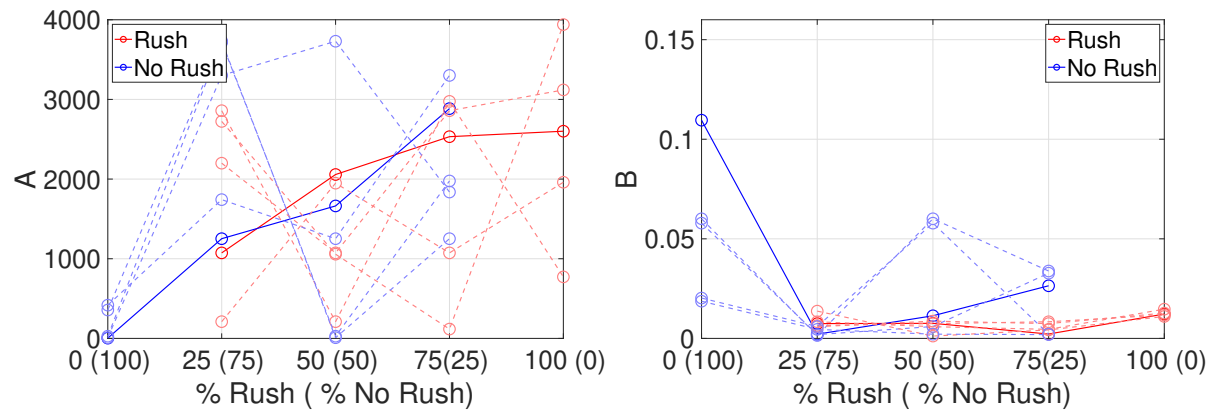

Figure 12 Top five fits for $A$ (left) and $B$ (right) parameters for all the conditions from the simulation. Blue shows the no-rush parameters ( $A_{\mathrm{NR}}$ in the plot on the left and $B_{\mathrm{NR}}$ in the plot on the right) while red shows the rush parameters ( $A_{\mathrm{R}}$ in the plot on the left and $B_{\mathrm{R}}$ in the plot on the right). The solid lines show the values for the best fitted parameters, while the dotted lines show the four next best.
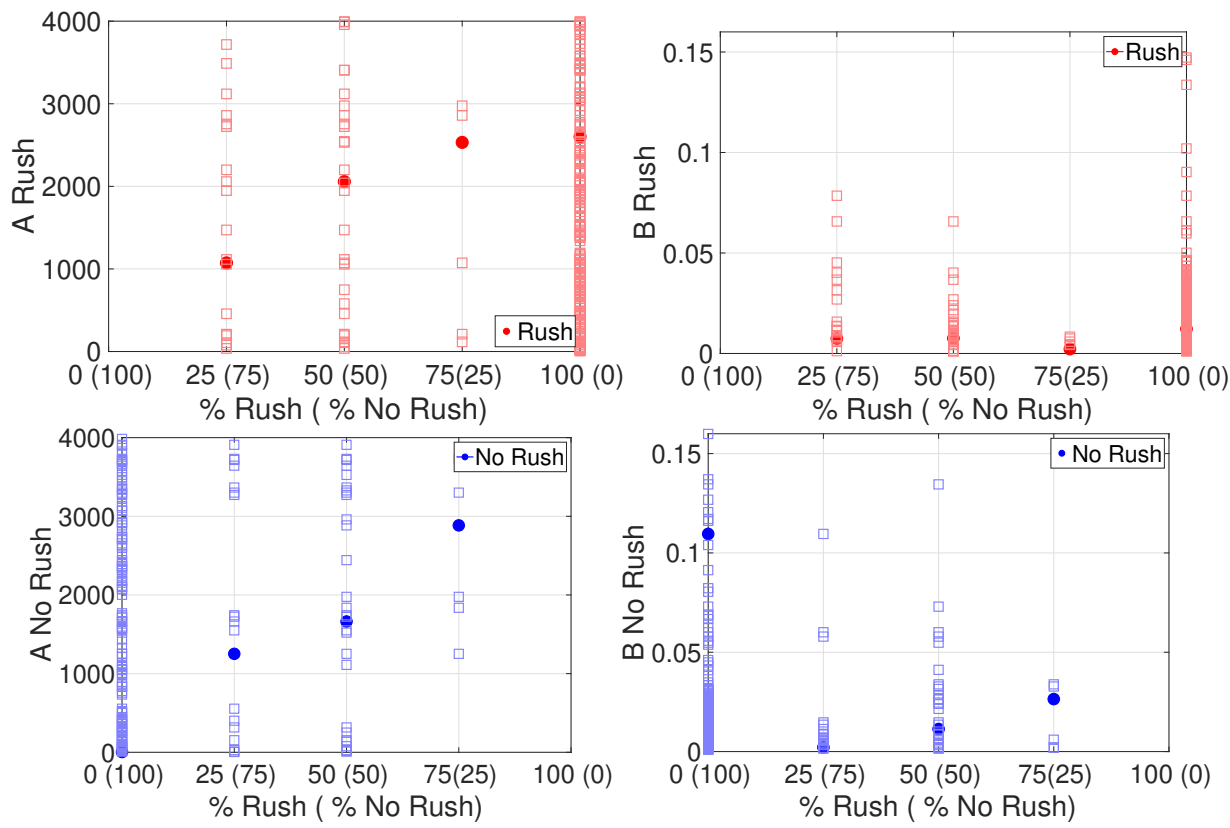

Figure 13 (Top Left) The values of parameter $A$ that are within a Wasserstein distance tolerance of 0.28 for all rush conditions from the simulation. The best $A$ is in a dark solid red circle, and the rest are in light red squares. (Top Right) The values of parameter $B$ that are within a Wasserstein distance tolerance of 0.28 for all rush conditions from the simulation. The best $B$ is plotted as a dark solid red circle. (Bottom Left) The values of parameter $A$ that are within a Wasserstein distance tolerance of 0.28 for all no-rush conditions from the simulation. The best value of $A$ is shown as a dark solid blue circle, and the rest are in light blue squares and the rest are in light red squares. (Bottom Right) The values of parameter $B$ that are within a Wasserstein distance tolerance of 0.28 for all no-rush conditions from the simulation. The best $B$ is shown as a dark solid blue circle, and the rest are in light blue squares. 
To compare the model more directly with the experimental results, we recreate several plots of the experimental results using one simulation with the best-fit parameters. In the bottom of Fig. 5, we plot the trajectories of the no rush agents in blue and the rush agents in red over each of the five conditions. Examining the figure, we observe that the simulated trajectories are quite smooth until they seemingly experience crowding at the door. At that point, the trajectories become much more chaotic, with the agents often temporarily heading away from the door, which we do not observe in the experimental plots at the top of the same figure.

In the bottom of Fig. 6 and the right plot of Fig. 7, we plot the analogue of the experimental data in the same plots. In the bottom of Fig. 6, we show the instantaneous speeds of the agents in one simulation of the model with the best-fit parameter values for each of the conditions, which can be found in Tab. 4. Individual agents' speeds are plotted in light colors, and the average instantaneous speeds are plotted in dark. There is a reasonable match of average instantaneous speed between the simulation and the experiment, see the top of the same figure. It is worth noting that while most of the experimental instantaneous speeds decrease over the course of the trial, most likely due to bottlenecks, we do not observe this pattern in our simulations, except in condition 0(100) where it is not observed in the experiments. We have also plotted, for reference, the desired speed $\left(v_{i}^{0}\right)$ for no rush agents as a dotted blue line, and the desired speed for the rush agents as a dotted red line; we can see a clustering of agents' individual speeds around these lines, particularly in conditions 0(100) and 100(0), where all of the agents are alike. On the right of Fig. 7, we plot the time to exit for all of the agents in one simulation of each condition, using the best-fit parameter values found in Tab. 4. As see in the experimental results plotted on the left of the same figure, we observe that the slowest exit time occurs for the condition with all no rush agents, and that there is a dip in the time to exit when several rush agents are added.

\section{Discussion}

Our experimental results point to the existence of social influence between people who would otherwise have very different tendencies to evacuate a room. Both rush and no-rush individuals tend to speed up when the other type is present, and rush individuals tend to deviate less from their shortest path if no-rush individuals are present. The source of this variation may be different for the rush and no-rush individuals, so we will consider them separately in the following two paragraphs.

For the no-rush individuals, we hypothesize that a few rush individuals create empty spots within the exiting crowd that no-rush individuals hurry to fill up. The tendency to fill such spots should manifest in the form of speed spikes in the instantaneous speeds, which we observe in the top of Fig. 5's 25(75) condition. Therefore, it is likely that the presence of rush individuals creates a sense of urgency among the no-rush individuals, leading the whole group to collectively evacuate faster than a purely no-rush one. It is also noteworthy that this effect is not amplified as the percentage of rush individuals increases within the group. This is likely because at half the crowd rushing, a huddle is formed quickly, which 
combined with the instruction of not to push, resulted in a general increase in evacuation time. This is evidenced in the top of Fig. 6, where the average instantaneous speeds match up for conditions 50(50) and 75(25) sooner than in 25(75). In light of the findings in [16], where the presence of hurried individuals in the crowd led to fast and slow phases of exit, it is possible that a comparatively smaller crowd as in our experiments experienced the onset of a slow phase. Note that we do not attribute the slowing down of rush individuals as their proportion within the group increased to the faster-is-slower effect, which even though has been shown to occur in many particle systems [25], was recently found to manifest only in the presence of pushing [26]. Similarly, it is worth commenting that the increased exit speed for no-rush individuals cannot be attributed to pushing from rush agents because they were instructed to not do so and we did not observe any such activity during the evacuation except when they huddled near the exit.

An equally interesting result is that the rush individuals speed up when there are norush individuals present. It is possible that the presence of no-rush individuals within the crowd allows the possibility of smooth flow through the exit for the rush individuals. We note this in the top of Fig. 5, where the red trajectories in 75(25) and 50(50) are less clumped together near the exit. Therefore, it is likely that physical forces played a bigger role than social influence in increasing the exit speed of rush individuals. A better flow for rush individuals may also explain the significant reduction in their deviation rate, when they found less obstructions in their path to exit. This is counter-intuitive in that we would expect the slower moving individuals to be perceived as obstacles by the faster moving individuals. However, it is likely that, for that to happen, the difference in speeds have to be significantly more than what we observed here. In other words, the results suggest that the presence of no-rush individuals simply provided clear exit paths to rush individuals without becoming obstacles.

Regarding our expectations, we did not see a clear dip followed by a rise in the time-toexit as the percentage of rush individuals grew within a group. This was likely due to the small crowd size in our experiment and the absence of pushing behavior. We did however find dependence of exit speeds of rush individuals (with respect to the presence of no-rush individuals) and vice versa. Even though we tested for and found a linear dependence, the average exit speeds suggest a nonlinear trend that should be tested with larger crowds.

Fitting the exit speeds to the social force model shows that the model is able to replicate the pattern of exit speeds with experimental condition, if we can vary the interaction parameters between different conditions. Allowing different parameters between conditions is equivalent to allowing individuals to change how they perceive and respond to their surroundings depending on the relative amounts of rush and no-rush individuals in the crowd. From a practical point of view, this seems like a reasonable adaptation for individuals in the crowd, and thus we allowed it in the model. Indeed, social factors have been shown to modulate risk perception during evacuations [13,27].

As a result, the fit of simulation and experimental data depends heavily on the values of $A$ and $B$, the pairwise interaction strength and characteristic length scale of the range, respectively. Patterns in the Wasserstein distance as a function of $A$ and $B$ can be observed in the plots in Tab. 5 beyond merely the location of the minimum distances. For example, a clear nonmonotonic trend in the Wasserstein distance can be tracked on curves 
from the lower left corner to upper right corner of the 0(100) and 25(75) no-rush plots. This suggests the presence of organization in the four-dimensional scalar field, whose dimensions are $A_{\mathrm{R}}, B_{\mathrm{R}}, A_{\mathrm{NR}}, B_{\mathrm{NR}}$, of which this is a two-dimensional slice. In short, the four parameters which determine the social interactions of rush and no-rush agents are not independent and thus must be viewed simultaneously when comparing with the experimental data. However, identifying such high-dimensional patterns are impossible to condense in low dimensions, which motivates the fitting process we used here.

When fitting the model parameters, it is critical that we understand the physical meaning of free parameters when comparing with experiments. The parameter $A$ multiplies the interaction force, which serves to weigh social interactions relative to physical forces such as the goal force or repulsive forces from walls. On the other hand, the parameter $B$ scales the range of social interactions, which defines a characteristic length within which social interactions occur. With this understanding, the best fit values of $A$ and $B$ provide insight into the mechanism by which the model is tuned to match the experiments. In particular, the best fit values of $A$ span the entire tested range of $A$, as can be seen in the left plots of Fig. 12 and 13. However, the best fit values of $B$ show decisive trends, which suggests that the model is insensitive to interaction strength but is sensitive to interaction range.

For both rush and no-rush individuals, the values of the best fit $B$ parameters show a clear increasing trend as the experiment moves from the pure conditions $0(100)$ and 100(0) to the conditions with mixed crowds. This means that, as individuals move with more individuals showing a different behavior, they interact with peers in increasingly larger physical ranges, which follows since we have seen that exit speeds increase in these cases as well. In other words, for more mixed groups, one must consider peers farther from oneself when planning exit paths, and then one is able to exit faster. These trends give insight into potential underlying mechanisms by which crowds generate different behaviors, namely that modifying their perception to consider larger peer neighborhoods may allow individuals to move more quickly [28]. Another way to perform the model fitting would be a statistical approach where model parameters that produce a matching distribution of an observable are considered as the best fit [29]. For example, this would amount to maximizing the posterior probability density of the observable conditioned on the parameters. In a Bayesian sense, however, this would entail selecting a prior distribution for the parameters $A$ and $B$.

Nevertheless, this interpretation of the social force model here reflects its highly restrictive nature in terms of the types of interaction forces which can occur, and their balancing using to Newton's second law. The challenges in fitting this model to the experimental data motivates the creation of a new model for crowd motion which is less constrained and may be able to better capture the compelling speed changes with experimental condition that we observe. Such a model will be developed in the future with this experimental data. 


\section{Acknowledgements}

Many thanks to Elham Mohammadi for her initial exploration of the model and to Daniel Balagué Guardia for his advice and expertise on programming matters. A.Ba's contribution to this research was supported in part by the National Science Foundation under Grant No. DMS-1319462. We would like to thank the anonymous reviewers for their detailed feedback and comments.

\section{References}

[1] Grosshandler, W.L., Bryner, N.P., Madrzykowski, D., Kuntz, K.: Draft report of the technical investigation of The Station nightclub fire. The Division (2005)

[2] Schadschneider, A., Klingsch, W., Klüpfel, H., Kretz, T., Rogsch, C., Seyfried, A.: Evacuation dynamics: Empirical results, modeling and applications. In: Meyers, R.A. (ed.) Encyclopedia of Complexity and Systems Science, pp. 3142-3176. Springer New York (2009)

[3] Zheng, X., Zhong, T., Liu, M.: Modeling crowd evacuation of a building based on seven methodological approaches. Building and Environment 44(3), 437-445 (2009). doi:10.1016/j.buildenv.2008.04.002

[4] Helbing, D., Farkas, I., Vicsek, T.: Simulating dynamical features of escape panic. Nature 407(6803), 487-90 (2000)

[5] Karamouzas, I., Skinner, B., Guy, S.J.: Universal power law governing pedestrian interactions. Physical Review Letters 113(23), 238701 (2014)

[6] Henein, C.M., White, T.: Agent-based modelling of forces in crowds. In: Multiagent and multi-agent-based simulation. Springer (2005)

[7] Yamamoto, K., Kokubo, S., Nishinari, K.: Simulation for pedestrian dynamics by real-coded cellular automata (RCA). Physica A: Statistical Mechanics and its Applications 379(2), 654-660 (2007)

[8] Pelechano, N., Allbeck, J.M., Badler, N.I.: Virtual crowds: Methods, simulation, and control. Synthesis Lectures on Computer Graphics and Animation 3(1), 1-176 (2008)

[9] Helbing, D., Farkas, I.J.: Simulation of pedestrian crowds in normal and evacuation situations. Pedestrian and Evacuation Dynamics 21, 21-58 (2002)

[10] Varas, A., Cornejo, M.D., Mainemer, D., Toledo, B., Rogan, J., Munoz, V., Valdivia, J.A.: Cellular automaton model for evacuation process with obstacles. Physica A: Statistical Mechanics and its Applications 382(2), 631-642 (2007) 
[11] Alizadeh, R.: A dynamic cellular automaton model for evacuation process with obstacles. Safety Science 49(2), 315-323 (2011)

[12] Daamen, W., Hoogendoorn, S.: Capacity of doors during evacuation conditions. Procedia Engineering 3, 53-66 (2010)

[13] Nilsson, D., Johansson, A.: Social influence during the initial phase of a fire evacuation-analysis of evacuation experiments in a cinema theatre. Fire Safety Journal 44(1), 71-79 (2009)

[14] Kinateder, M., Warren, W.H.: Social influence on evacuation behavior in real and virtual environments. Frontiers in Robotics and AI 3, 43 (2016)

[15] Heliövaara, S., Ehtamo, H., Helbing, D., Korhonen, T.: Patient and impatient pedestrians in a spatial game for egress congestion. Physical Review E 87(1), 012802 (2013)

[16] Nicolas, A., Bouzat, S., Kuperman, M.N.: Pedestrian flows through a narrow doorway: Effect of individual behaviours on the global flow and microscopic dynamics. Transportation Research Part B: Methodological 99, 30-43 (2017)

[17] Deaton, A.: Height, health, and inequality: the distribution of adult heights in india. American Economic Review 98(2), 468-74 (2008)

[18] Butail, S., Bartolini, T., Porfiri, M.: Collective response of zebrafish shoals to a free-swimming robotic fish. PLoS One 8(10), e76123 (2013). doi:10.1371/journal.pone.0076123

[19] Johansson, F., Duives, D., Daamen, W., Hoogendoorn, S.: The many roles of the relaxation time parameter in force based models of pedestrian dynamics. Transportation Research Procedia 2, 300-308 (2014)

[20] Dewangan, K.N., Owary, C., Datta, R.K.: Anthropometry of male agricultural workers of north-eastern India and its use in design of agricultural tools and equipment. International Journal of Industrial Ergonomics 40(5), 560-573 (2010)

[21] Santner, T.J., Williams, B.J., Notz, W., Williams, B.J.: The design and analysis of computer experiments. Springer (2003)

[22] Villani, C.: Topics in optimal transportation. 58. American Mathematical Soc. (2003)

[23] Carrillo, J.A., Toscani, G.: Wasserstein metric and large-time asymptotics of nonlinear diffusion equations. In: New Trends in Mathematical Physics: In Honour of the Salvatore Rionero 70th Birthday, pp. 234-244. World Scientific (2004)

[24] Peyre, G., Cuturi, M.: Computational optimal transport. Foundations and Trends in Machine Learning 11(5-6), 355-607 (2019) 
[25] Pastor, J.M., Garcimartín, A., Gago, P.A., Peralta, J.P., Martín-Gómez, C., Ferrer, L.M., Maza, D., Parisi, D.R., Pugnaloni, L.A., Zuriguel, I.: Experimental proof of faster-is-slower in systems of frictional particles flowing through constrictions. Physical Review E 92(6), 062817 (2015)

[26] Haghani, M., Sarvi, M., Shahhoseini, Z.: When 'push'does not come to 'shove': Revisiting 'faster is slower'in collective egress of human crowds. Transportation Research Part A: Policy and Practice 122, 51-69 (2019)

[27] Siegrist, M., Cvetkovich, G.: Perception of hazards: The role of social trust and knowledge. Risk Analysis 20(5), 713-720 (2000)

[28] Smith, A.: Contribution of perceptions in analysis of walking behavior. Transportation Research Record 2140(1), 128-136 (2009)

[29] Bode, N.W.F., Ronchi, E.: Statistical model fitting and model selection in pedestrian dynamics research. Collective Dynamics 4, 1-32 (2019) 